\title{
Sinus surgery postpones chronic Gram-negative lung infection: cohort study of 106 patients with cystic fibrosis*
}

\section{Mikkel Christian Alanin'1, Kasper Aanaes', Niels Høiby',3, Tania Pressler4, Marianne Skov ${ }^{4}$, Kim G. Nielsen ${ }^{4,5}$, David Taylor-Robinson ${ }^{6}$, Elisabeth Waldmann ${ }^{7}$, Helle Krogh Johansen², Christian von Buchwald ${ }^{1}$}

'Department of Otorhinolaryngology, Head and Neck Surgery and Audiology, Rigshospitalet, Denmark

2 Department of Clinical Microbiology, Rigshospitalet, Denmark

${ }^{3}$ Institute of Immunology and Microbiology, University of Copenhagen, Denmark

${ }^{4}$ Copenhagen CF Centre, Rigshospitalet, Denmark

SPaediatric Pulmonary Service, Department of Paediatrics and Adolescent Medicine, Rigshospitalet, Denmark

${ }^{6}$ Department of Public Health and Policy, University of Liverpool, United Kingdom

Department of Medical Informatics, Biometry and Epidemiology, Friedrich-Alexander-Universität Erlangen-Nürnberg, Germany
Rhinology 54: 206-213, 2016

DOl:10.4193/Rhino15.347

*Received for publication:

November 27, 2015

Accepted: February 27, 2016

\begin{abstract}
Background: In patients with cystic fibrosis (CF) the sinuses are a bacterial reservoir for Gram-negative bacteria (GNB). From the sinuses the GNB can repeatedly migrate to the lungs. In a one-year follow-up study, endoscopic sinus surgery (ESS) with adjuvant therapy reduced the frequency of pulmonary samples positive for GNB. We investigated whether the effect is sustained.
\end{abstract}

Methodology: We report the effect of ESS and adjuvant therapy three years postoperatively in a CF cohort participating in this prospective clinical follow-up study. The primary endpoint was the lung infection status defined by Leeds criteria.

Results: One hundred and six CF patients underwent ESS; 27 had improved lung infection status after three years. The prevalence of patients free of lung colonization with GNB significantly increased from 16/106 patients (15\%) preoperatively to 35/106 patients (33\%) after three years. The total cohort had decreasing lung function during follow-up; however, in 27 patients with improved lung infection status lung function was stable. Revision surgery was performed in 31 patients (28\%).

Conclusion: ESS with adjuvant therapy significantly improves the lung infection status for at least three years in our cohort of patients with CF and may postpone chronic lung infection with GNB and thus stabilize lung function.

Key words: lower airway cultures, Pseudomonas aeruginosa, quality of life, pulmonary function, ESS

\section{Introduction}

In patients with cystic fibrosis (CF) the mucociliary escalator is compromised due to a defect in chloride ion transport leading to depletion of the periciliary liquid layer ${ }^{(1)}$ and impaired mucus detachment ${ }^{(2)}$. The result is viscous mucus and decreased mucociliary clearance ${ }^{(3)}$.

Morbidity and mortality in CF are significantly influenced by pulmonary colonization and infections with particularly Gram-negative bacteria (GNB) such as Pseudomonas aeruginosa, Achromobacter xylosoxidans, and Burkholderia spp.; thus intensive antibiotic treatment is mandatory ${ }^{(4)}$. Patients are usually intermittently lung colonized in childhood, but despite intensive antibiotic therapy chronic infection is rarely prevented - only postponed. Approximately $32 \%$ of all Danish CF patients are chronically infected in early adulthood ${ }^{(5)}$. The bacteria in the lungs are initially susceptible to antibiotics ${ }^{(6)}$ and can usually be eradicated in intermittently colonized patients. Nevertheless, a patient is often re-colonized with the identical genotype ${ }^{(7,8)}$. The source of this re-colonization can be the paranasal sinuses, which may act as a bacterial reservoir ${ }^{(7,9-12)}$, where the GNB can adapt and repeatedly migrate to and colonize the lungs ${ }^{(13)}$. Similar source of re-colonization applies in lung transplanted CF 
patients causing lung allograft infections ${ }^{(14,15)}$.

Chronic rhinosinusitis (CRS) with or without nasal polyposis is common in patients with $\mathrm{CF}^{(16,17)}$, and has a substantial negative impact on quality of life ${ }^{(18)}$. No international guidelines for standardised therapeutic strategy in CRS management or management of sinus infections in CF currently exist and systemic antibiotic treatment may only have a limited effect on upper airway bacterial infections in CF encouraging surgical management ${ }^{(19)}$.

We have previously demonstrated a significant one year postoperative effect on the frequency of pulmonary samples positive for GNB, sinus symptoms measured by Sino nasal outcome test 22 (SNOT-22) ${ }^{(20)}$, and a general improved health related quality of life (HR-QoL) (21) in 106 patients with CF ${ }^{(22)}$. In the present study we have extended the follow-up period with additional two years in this well characterized cohort.

The primary aim was to investigate longer-term impacts of ESS on CF outcomes, and specifically to understand if ESS with adjuvant therapy affects the lung infection status, and the lung function decline in CF patients intermittently lung colonized with $P$. aeruginosa.

\section{Materials and methods}

\section{Patients}

We undertook a prospective, uncontrolled, observational study of 106 CF patients followed at the CF Centre Copenhagen, and who had ESS in the period from September 2007 to January 2012. Consequently, all patients had a minimum of three years follow-up in January 2015 with outcome data on lower airway samples, antibody response against GNB in serum samples, body weight, lung function tests, and quality of life-questionnaires. Post ESS data were compared with preoperative data extracted from our CF database.

CF patients affiliated with the outpatient clinic of the CF Centre Copenhagen are seen on a monthly basis for clinical examination, lung function measurement, and collection of sputum sample for microbiological culture. Blood samples are obtained every third month for analyses.

Besides ESS with adjuvant therapy the daily treatment protocol was unchanged during the period studied ${ }^{(23)}$. The CF diagnosis, demographic data, the indication for ESS and the results one year after ESS have been reported previously ${ }^{(22)}$. In brief, the three main indications for ESS are:

- Search for an infectious focus in CF patients intermittently lung colonized with GNB and/or increase in precipitating antibodies

- Patients with recent (with-in one year) lung transplantation

- Patients with severe symptoms of CRS according to the European position paper on rhinosinusitis and nasal polyps (EPOS) criteria ${ }^{(24)}$ with unsatisfactory effect of conservative treatment.
Endoscopic sinus surgery and adjuvant therapy ESS was performed with a navigation system based on a preoperative computed tomography scan with at least $1 \mathrm{~mm}$ slice thickness and included uncinectomy, anterior anthrostomy with creation of large openings comprising more than half the medial maxillary wall, ethmoidectomi, and frontal- and sphenoid sinus anthrostomies. Multiple samples for culture were obtained. Postoperative adjuvant therapy include two weeks of intravenous antibiotics according to susceptibility testing of the cultured GNB, twice daily nasal irrigations with saline and local antibiotics adjusted after susceptibility testing for six months, topical nasal steroids for at least three months, and rhinologic follow-up after 14 days, three, six and 12 months ${ }^{(22)}$.

\section{Lung infection status}

Leeds criteria ${ }^{(25)}$ were used to define GNB infection status:

- chronic infection, when $>50 \%$ of the preceding 12 months' sputum cultures were positive

- intermittent infection, when $\leq 50 \%$ of the preceding 12 months' sputum cultures were positive

- free from infection, no growth of bacteria in the lungs within the previous 12 months

We compared the GNB lung infection status two and three years after ESS with the status prior to ESS.

\section{Lower airway cultures}

The percentage of positive lower airway samples with CF pathogens 365 days preceding ESS were compared with cultures obtained 365-1095 days following ESS. We did not discriminate between outcomes of the methods used to obtain the sample: expectorated sputum, endolaryngeal suction or bronchoalveolar lavage (BAL). However, using microscopy on all samples it was ensured that lower airways were represented.

Only the most frequently cultured GNB was evaluated. Our main interest was the frequency of positive bacterial samples with the dominant pathogen from the lower airways before and after ESS. If patients were colonized with a new CF pathogen after initial eradication it was recorded and reported. Sinus and lower airway samples were cultured as previously described ${ }^{(26)}$.

\section{Lung function tests}

Spirometry was performed at every visit before ESS and at every visit during follow-up. We report the average of all measurements as percent of predicted values and as trend slopes the year before surgery, and two and three years after as described previously ${ }^{(22)}$. We hypothesized, that ESS with adjuvant therapy would reduce the decline in lung function in patients intermittently colonized with P. aeruginosa, and assessed the effect of ESS on the mean \%FEV1 trajectory over time. The methods are described in (27). 


\section{Body Mass Index}

Height and weight were recorded and body mass index (BMI z-scores) calculated at every visit. The mean BMI prior to surgery was compared with postoperative values.

\section{Sino nasal outcome test 22 (SNOT-22) and Health-related quality of life (HRQoL)}

SNOT-22 is a validated health related quality of life instrument. It highlights the impact of chronic rhinosinusitis on quality of life, and can also be used to estimate the effect of ESS ${ }^{(20)}$. The Cystic Fibrosis Questionnaire health-related quality of life (HRQOL) is a validated disease-specific instrument that measures health-related quality of life that can be utilized in clinical trials to assess the effects of new therapies ${ }^{(21)}$.

Questionnaires collected at least three years postoperatively were compared with pre-operative scores from patients who had been evaluated previously ${ }^{(22)}$. Two age-related HRQoL questionnaires exist: one designed for patients below 14 years, and one for patients above 14 years of age. Patients completed the same age-related questionnaire as before surgery independent of their postoperative age for comparison.

\section{Serum GNB specific antibodies}

Precipitins against relevant CF GNB using crossed immunoelectrophoresis are measured annually in CF patients. Normal values are 0 and 1 . Two or more precipitins against $P$. aeruginos $a$ are considered as chronic infection ${ }^{(28,29)}$.

\section{Pulsed-field gel electrophoresis (PFGE)}

Stored $P$. aeruginosa isolates from patients with 1 ) intermittent lung colonization at the time of surgery, 2) a positive per operative sinus culture, and 3 ) intermittent recolonization with P. aeruginosa after ESS were genotyped using PFGE as described previously ${ }^{(26)}$. The PFGE bands were evaluated visually according to the guidelines of Tenover et al. ${ }^{\left({ }^{30}\right)}$. Isolates with differences in two or less bands were considered identical. We assessed the relatedness between isolates obtained during ESS with the recolonizing isolate.

\section{Statistics}

Statistical Software Analysis (SAS), version 9.3 was used for statistical analysis comparing pre- and postoperative measurements. A paired t-test was used to compare the measured data. McNemar's test was used to compare the change in lung infection status.

\section{Ethics}

The study was approved by the Danish Data Protection Agency (2014-41-3497).

\begin{tabular}{|c|c|c|c|}
\hline $\begin{array}{l}\text { Lung infection } \\
\text { status at ESS }\end{array}$ & & $\begin{array}{l}\text { Lung infection } \\
\text { status one year } \\
\text { after ESS } \\
(22)\end{array}$ & $\begin{array}{l}\text { Lung infection } \\
\text { status three } \\
\text { years after ESS }\end{array}$ \\
\hline CF - / $16(15 \%)$ & $\begin{array}{l}\nearrow \\
\rightarrow \\
\searrow\end{array}$ & $\begin{array}{l}C F-\quad / 14(13 \%) \\
C F+(i) / 2(2 \%) \\
C F+(c) / 0(0 \%)\end{array}$ & $\begin{array}{l}\mathrm{CF}-\quad / 12(11 \%) \\
\mathrm{CF}+(\mathrm{i}) / 2(2 \%) \\
\mathrm{CF}+(\mathrm{c}) / 2(2 \%)\end{array}$ \\
\hline$C F+(i) / 61(58 \%)$ & $\begin{array}{l}\lambda \\
\rightarrow \\
y\end{array}$ & $\begin{array}{l}C F-\quad / 22(21 \%) \\
C F+(i) / 35(33 \%) \\
C F+(c) / 4(4 \%)\end{array}$ & $\begin{array}{l}C F-\quad / 22(21 \%) \\
C F+(i) / 28(26 \%) \\
C F+(c) / 11(10 \%)\end{array}$ \\
\hline$C F+(c) / 29(27 \%)$ & $\begin{array}{l}\lambda \\
\rightarrow \\
\searrow\end{array}$ & $\begin{array}{l}C F-\quad / 4(4 \%) \\
C F+(i) / 1(1 \%) \\
C F+(c) / 24(23 \%)\end{array}$ & $\begin{array}{l}C F-\quad / 1(1 \%) \\
C F+(i) / 4(4 \%) \\
C F+(c) / 24(23 \%)\end{array}$ \\
\hline
\end{tabular}

Figure 1. Infection status before, one year after 22, and three years after endoscopic sinus surgery (ESS). Non-colonized patients (CF-). Intermittently colonized patients (CF+ (i)). Chronically infected patients $(C F+(c))$. The number of non-colonized patients more than doubled from $16(15 \%)$ to 35 (33\%) three years after combined endoscopic sinus surgery (ESS) and adjuvant therapy including continuously active management of the upper airways. The number of chronically infected patients increased from $29(27 \%)$ to 37 (35\%) and the number of intermittently colonized patients decreased from 61 (58\%) to 34 (32\%). Overall, the groups differed significantly before and three years after ESS, $p<0.001$. Details about the results one year after ESS is reported previously ${ }^{(22)}$.

\section{Results}

Patients

ESS was performed in 106 patients with CF, (mean age 19 years, range 6-50 years). Double LTX had been performed in 15 patients (14\%) prior to surgery. Six patients (6\%) died (without relation to ESS) during follow-up but all later than three years postoperatively allowing clinical follow-up data. However, data from questionnaires were not available for all patients. Revision ESS was performed in 31 patients (29\%) within three years and three patients had revision surgery twice. The median time to revision surgery was 22 months (range $8-35$ months). The indication for revision surgery was severe symptoms of CRS in 20 patients and search for an infectious focus and subsequent eradication attempt in 11 patients.

\section{Lung infection status}

The number of non-colonized patients (CF-) more than doubled from $16(15 \%)$ at the time of surgery to $35(33 \%)$ three years after sinus surgery. The number of intermittently colonized patients (CF+ (i)) decreased over three years period from 61 (58\%) to $34(32 \%)$ and the number of chronically infected patients (CF+ (c)) increased from 29 (27\%) to 37 (35\%). Overall 27 pa- 


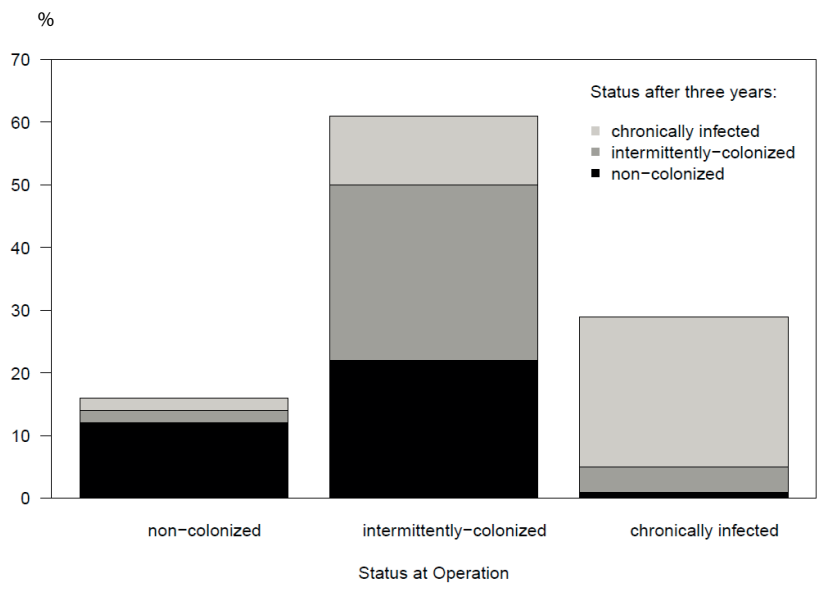

Figure 2. Illustration of the lung infection status before and three years after ESS in a cohort of 106 patients with cystic fibrosis. The groups differed significantly before and after endoscopic sinus surgery, $\mathrm{p}<0.001$.

tients improved in lung infection status, Figure 1 and the groups differed significantly before and after ESS, $\mathrm{p}<0.001$ (Figure 2). In total 13 out of 106 patients (12\%) became chronically infected during the three year follow-up. Noticeable, six of the 13 patients (46\%) had elevated precipitins against the dominant GNB before ESS. Changes in lung infection status during the three-year follow-up are illustrated in Figure 3. Temporarily or persistent change in lung infection status in relation to the status before ESS with adjuvant therapy was observed in six out of $16(38 \%)$ patients free of infection, in 47 out of 61 (77\%) intermittently colonized patients and in five out of 29 (17\%) patients with chronic infection.

\section{Lower airway cultures}

The mean percentages of pre- and postoperative lower airway cultures with growth of CF GNB are presented in Table 1. The total cohort had a tendency towards fewer culture positive samples. In subgroup analyses there was a statistically significant reduction in positive lower respiratory tract cultures in patients non- or intermittently colonized (CF- and CF+ (i), n=76), $\mathrm{P}=0.002$ (Table 2a).

Lung function tests and longitudinal analysis of FEV1 trajectory We observed decreasing lung function during follow-up, Table 2. The FEV1\% and FVC\% predicted value decline was statistically significant but without difference in lung function slopes preand postoperatively. In patients intermittently colonized with $P$. aeruginosa we performed longitudinal analyses comparing the lung function trajectory with CF patients from the Copenhagen cohort not undergoing ESS.

The longitudinal dataset included 21,658 lung function measurements on 178 patients measured between 1984 and 2014 at the CF Centre Copenhagen. Seventy-four patients underwent ESS. Date of surgery was not associated with a significant

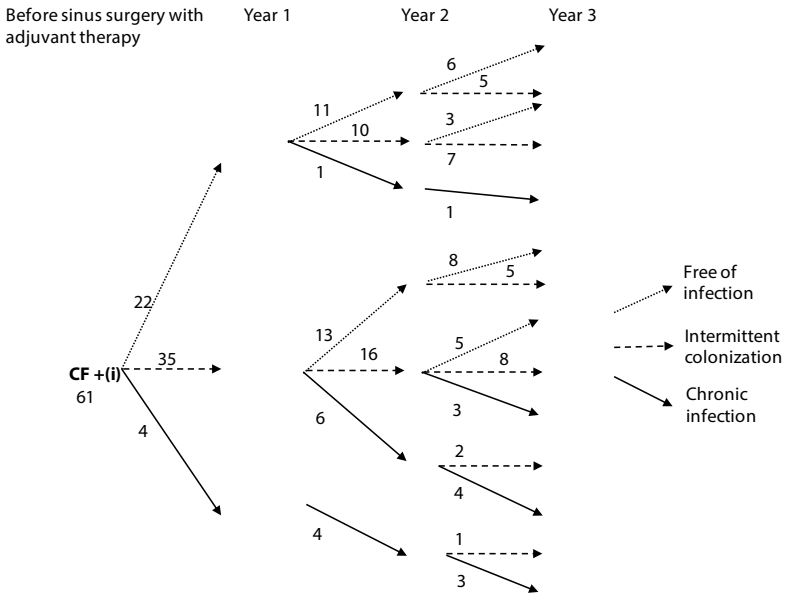

Figure 3. Flow-chart illustrating the change in lung infection during the three-year follow-up in the 61 patients with intermittent lung colonization. Before endoscopic sinus surgery with adjuvant therapy 61 patients were intermittently lung colonized with Gram-negative bacteria (GNB) where a change in lung infection status was most frequently observed during follow-up. Patients were classified according to the Leeds criteria. Temporarily or persistent change in lung infection status was observed in 47 out of $61(77 \%)$ patients. The course of infection is displayed with arrows.

change in the rate of decline in \%FEV1 after adjustment for demographic, genetic and clinical factors. In the 27 patients with improved lung infection status after three years we observed stable lung function, mean FEV1\% was 96 before surgery with adjuvant therapy and 95 after, $\mathrm{p}=0.5$.

The results from body mass index (BMI) and, , precipitins are shown in Table 1, and the results from lung function before and after ESS are presented in Table 2.

\section{Questionnaires}

Pre-operative scores in SNOT 22 and HRQoL were completed by $86(87 \%)$ and 67 (78\%) patients respectively. Six patients (6\%) died during follow-up therefore questionnaires could not be obtained.

\section{SNOT 22}

We observed a decrease (an improvement) on sinonasal symptoms following ESS after a median follow-up of 52 months; however, the decrease was not significant, Table 3.

\section{Health-related quality of life (HRQOL)}

An significant decrease (a worsening) was observed in adult patients after a median of 52 months follow-up, $p=0.04$. No change was observed in children, Table 3.

\section{Pulsed-field gel electrophoresis (PFGE)}

We genotyped isolates of $P$. aeruginosa from 31 patients who had a combination of intermittent lung colonization with $P$. 
Table 1. Pre- and post ESS clinical data from 106 CF patients two and three years after surgery.

\begin{tabular}{|c|c|c|c|c|}
\hline $\begin{array}{l}\text { CF patients (n) } \\
\text { One year prior to ESS / } \\
\text { two years after ESS / } \\
\text { three years after ESS }\end{array}$ & $\begin{array}{l}\text { Intermittently colonized } \\
\text { with Gram-negative } \\
\text { bacteria } \\
\text { (61) }\end{array}$ & $\begin{array}{l}\text { Non-infected }+ \\
\text { intermittently } \\
\text { colonized } \\
(16+61=77)\end{array}$ & $\begin{array}{l}\text { Chronically infected } \\
\text { (29) }\end{array}$ & $\begin{array}{l}\text { Total } \\
(106)\end{array}$ \\
\hline $\begin{array}{l}\text { Mean number of lung function tests } \\
\text { and z-BMI }\end{array}$ & $13 / 11 / 10$ & $12 / 11 / 10$ & $10 / 10 / 10$ & $12 / 10 / 10$ \\
\hline Mean\% positive airway cultures & $24 / 23 / 31$ & $18 / 16 / 14 *$ & $87 / 95 / 74^{*}$ & $38 / 37 / 38$ \\
\hline Mean z-BMI & $-0.1 /-0.2 /-0.2$ & $-0.2 /-0.3 /-0.3$ & $-0.5 /-0.6 /-0.7$ & $-0.3 /-0.4^{*} /-0.4^{*}$ \\
\hline Mean Precipitins against GNB & $2.4 / 3.5^{*} / 4.7^{*}$ & $2.2 / 3.2^{*} / 4.2^{*}$ & $10.7 / 13.5^{*} / 14.5^{*}$ & $3.0 / 5.8^{*} / 6.9^{*}$ \\
\hline
\end{tabular}

${ }^{*} \mathrm{p}<0.05$. Pairwise comparison (t-test) with preoperative clinical data.

Table 2. Pre- and post ESS lung function data from 106 CF patients two and three years after surgery.

\begin{tabular}{|c|c|c|c|c|}
\hline $\begin{array}{l}\text { CF patients (n) } \\
\text { One year prior to ESS / } \\
\text { two years after ESS / } \\
\text { three years after ESS }\end{array}$ & $\begin{array}{l}\text { Intermittently colonized } \\
\text { with Gram-negative } \\
\text { bacteria } \\
\text { (61) }\end{array}$ & $\begin{array}{l}\text { Non-infected + } \\
\text { intermittently } \\
\text { colonized } \\
(16+61=77)\end{array}$ & $\begin{array}{l}\text { Chronically infected } \\
\text { (29) }\end{array}$ & $\begin{array}{l}\text { Total } \\
(106)\end{array}$ \\
\hline Mean FEV1\% & $85 / 80 * / 79 *$ & $85 / 81 * / 79 *$ & $69 / 65 / 63$ & $81 / 77^{*} / 75^{*}$ \\
\hline Mean FEV1 slope & $-1.8 /-3.8 /-4.2$ & $-3.1 /-4.0 /-4.9$ & $1.6 /-2 /-1.9$ & $38 / 37 / 38$ \\
\hline Mean FVC\% & $-0.1 /-0.2 /-0.2$ & $-0.2 /-0.3 /-0.3$ & $83 / 82 / 80$ & $92 / 90^{*} / 88^{*}$ \\
\hline Mean FVC slope & $-0.7 /-2.2 /-3.3$ & $-1.4 /-2.2 /-3.9$ & $0.4 /-2.2 /-1.7$ & $-1.0 /-2.2 /-3.4$ \\
\hline
\end{tabular}

${ }^{*} \mathrm{p}<0.05$. Pairwise comparison (t-test) with preoperative clinical data.

Table 3. Results of the questionnaires before and at least three years after ESS.

\begin{tabular}{|c|c|c|}
\hline & Pre-operatively & $>3$ years \\
\hline Number of patients who completed SNOT-22, (response rate), [median time after surgery] & 86 & 75 (87\%), [53 months] \\
\hline Mean SNOT-22 (range) & $17(0-76)$ & $14.5(0-84)$ \\
\hline Number of patients who completed CFQ-R, (response rate), [median time after surgery] & 67 & $52(78 \%),[52$ months] \\
\hline \multicolumn{3}{|l|}{ Mean (CFQ-R) (range) } \\
\hline $6-13$ years & $143(113-170)$ & $143(111-168)$ \\
\hline$>13$ years & $160(101-188)$ & $154 *(112-191)$ \\
\hline
\end{tabular}

${ }^{*} \mathrm{p}<0.05$. Pairwise comparison (t-test) with preoperative clinical data.

aeruginosa, peroperatively positive sinus culture with $P$. aeruginosa, and recolonization after ESS with $P$. aeruginosa. Ten out of 31 patients (32\%) were recolonized with a new clone type and in the remaining $68 \%$ patients were recolonized with the same identical clone type.

\section{Change in dominant lung pathogen}

A shift in the dominant lung pathogen after ESS with adjuvant therapy was observed in six patients (6\%); three patients, intermittently colonized with $P$. aeruginosa preoperatively, changed to A. xylosoxidans and one patient eventually became chronically infected. One patient changed to chronic infection with Burkholderia multivorans. The mean period until change was 600 days. The reverse infection pattern (from A. xylosoxidans to intermittent $P$. aeruginosa infection) was true in two patients after a mean of 726 days.

\section{Discussion}

This study comprises the largest prospective study with the longest follow-up assessing the effect of ESS in CF patients. It was conducted to determine the long-term effects of combined ESS, adjuvant therapy and continuously active management of the 
upper airways on microbial pathogens, lung function trajectory and self-reported sinus symptoms and HRQoL in patients with CF. The significant short-term (one year follow-up) results on the identical cohort are reported previously ${ }^{(22)}$.

Despite the natural disease progression seen in CF patients, our results demonstrate that our newly introduced treatment regime, consisting of ESS with adjuvant therapy, improves lung infection status, reduces the frequency of pulmonary samples positive for GNB for a period of at least three years, and hence may postpone chronic lung infection especially in $\mathrm{CF}$ patients with intermittent lung colonization with GNB, where a change in lung infection status is most frequently observed (77\%). Most importantly, our results demonstrate that when this treatment regime successfully eradicates upper and lower airway pathogens and postpones chronic lung infection status on the longterm, lung function can be stabilized for at least three years. Furthermore, a change in the dominant lung GNB after ESS was uncommon. We encourage close multidisciplinary collaboration between clinical microbiologists, paediatric pulmonologists and otorhinolaryngologists for optimal management of these patients.

CRS has a negative impact on QoL both in the general population ${ }^{(24)}$ and in patients with $\mathrm{CF}^{(18)}$. Our previous results ${ }^{(22)}$ demonstrated that CF patients benefit significantly from ESS with regard to sinus symptoms and HRQoL on the short-term, which is in agreement with the study by Taylor et al. ${ }^{(31)}$. However, the effect did not last three to four years in spite of revision surgery in approximately one third of the patients. A possible explanation may be that CF patients tend to underreport their sinus symptoms ${ }^{(10,32)}$ before ESS but when symptoms of CRS eventually recur the patients may be more aware of these and may find them more severe causing a greater impact of sinus symptom score than previously. Nevertheless, ESS has proved to be effective on the long-term in children, including CF children, with CRS with nasal polyps ${ }^{(33)}$.

Also in non-CF patients with CRS with polyposis revision surgery is common. Until better treatment modalities are found, we believe that several CF patients are in need of revision surgery. ESS is a safe procedure ${ }^{(24)}$ and it should be the recurrence of severe symptoms of CRS that sets the date for revision, regardless if the timespan is months or years.

The indications for ESS varied within the cohort: search for an infectious focus or severe symptoms of CRS. An interesting subgroup is patients intermittently lung colonized with $P$. aeruginosa where the surgical indication is search for an infectious focus and subsequent eradication attempt. The majority of these patients were on the border of having chronic lung infection with elevated antibodies against GNB, which is associated with a rapid declining lung function and poor prognosis
(34). We hypothesized that combined ESS and adjuvant therapy positively affects the lung function trajectory. However, ESS was not associated with a reduction in lung function decline, which is in agreement with previous prospective studies ${ }^{(35,36)}$. The fact that this specific cohort had a good preoperative lung function with a mean FEV1 of $85 \%$ in all patients not chronically infected and that 12 patients (11\%) had a preoperative FEV1 above $100 \%$ also suggest that it is indeed hard to improve lung function from such a starting point.

The strength of the longitudinal lung function analysis is the high frequency follow-up in this unique Danish dataset that has allowed us to fit a more sophisticated model, which we believe leads to more efficient estimation of the rate of lung function decline in the CF Center, Copenhagen (27).

Our study has limitations. First, we did not perform a randomized controlled study initially. However, we may now consider such a study of ethical controversy since eradication of GNB from the sinuses and lungs have been successful and longlasting (>3 years) in several cases. Further, the presented results may underestimate the effect of ESS with adjuvant therapy: in $32 \%$ of cases patients were recolonized with a new clone type after ESS indicating that the primary eradication attempt was successful, which would only strengthen our results and conclusions. Further, a number of the intermittently lung colonized patients may have progressed to chronic lung infection shortly before ESS. We would have identified six of these if we had combined the Leeds criteria for assessing the lung infection status ${ }^{(25)}$ with the results of serum precipitating antibodies ${ }^{(37)}$. Eradication is very rare in chronically lung infected patients 8 and they have a steeper annual decline in lung function ${ }^{(34)}$. However, serum precipitating antibodies were not used in the present study to classify patients, which was intentionally to make it more comparable to other studies and centers. In addition, even though microscopy was used on all respiratory samples to verify lower airway representation, contamination from the upper airway may have occurred, which may represent a limitation.

Other studies, mainly retrospective and only including few patients have assessed the effect of sinus surgery in CF with inconsistent results, and to date there is no standardised therapeutic strategy for CRS management or management of sinus infections in $\mathrm{CF}^{\left({ }^{10}\right)}$. However, our experience supported by other prospective studies using a protocolled postoperative regimen ${ }^{(15,35,38)}$ indicate that combined ESS and adjuvant therapy promotes the best outcome both concerning CRS management and in reducing airway colonization with GNB; however, revision surgeries may be required.

The observation that combined ESS and adjuvant therapy may postpone chronic lung infection in intermittently colonized patients encourages the development of methods to identify early lung infections or preferably patients with GNB in the sinuses ${ }^{(22)}$. We encourage ESS and adjuvant therapy as soon as possible 
after initial colonization with GNB. Precipitating antibodies and measurements of anti-pseudomonal secretory immunoglobulin $A(\lg A)$ in nasal secretions or saliva may prove to be effective to identify these patients ${ }^{(39)}$.

The findings from this study have a number of clinical applications that can recommend ESS with adjuvant therapy in CF since it can postpone the chronic lung infection status in patients intermittently colonized on the long-term (at least three years) and improve sinus symptoms in CF patients on the short-term.

\section{Conclusion}

The conclusion from the present study may be inspirable and transferable to other patients with diseases affecting the mucociliary apparatus such as patients with primary ciliary dyskinesia. In these patients simultaneous sinus and lung infections with identical pathogens have been reported ${ }^{(40)}$. However, large scale randomised studies with standardised methods are needed to determine the exact role of ESS in CF, which are currently being planned.

\section{Acknowledgements}

We thank laboratory technicians Ulla Rydal Johansen, Lena Nørregaard, Katja Bloksted (Department of Clinical Microbiology) for their dedication to the project. The Candys Foundation, a non-profit organization, supported MCA as a PhD stipend. The Novo Nordisk Foundation supported HKJ as a clinical research stipend. KGN received funding from the European Union Seventh Framework Programme (FP7/2007-2013) under grant agreement n8305404 (BESTCILIA).

\section{Authorship contribution}

All authors contributed to the conception of this work, revised it critically for important intellectual content, interpreted the data, and approved the final version to be published. MCA acquired and analysed data and drafted the work. DTR and EW performed the longitudinal analysis of FEV1 trajectory.

\section{Conflict of interest}

The authors have no conflicts of interest to declare pertaining to this article.

\section{References}

1. Boucher RC. New concepts of the pathogenesis of cystic fibrosis lung disease. Eur Respir J 2004;23(1):146-58.

2. Hoegger MJ, Fischer AJ, McMenimen JD, et al. Impaired mucus detachment disrupts mucociliary transport in a piglet model of cystic fibrosis. Science 2014;345(6198):818 22.

3. Matsui H, Grubb BR, Tarran R, et al. Evidence for periciliary liquid layer depletion, not abnormal ion composition, in the pathogenesis of cystic fibrosis airways disease. Cell 1998; 95(7):1005-15.

4. Gibson RL, Burns JL, Ramsey BW Pathophysiology and management of pulmonary infections in cystic fibrosis. Am J Respir Crit Care Med 2003;168(8):918-51.

5. Zolin A, Mckone EF, van Rens J. ECFS patient registry 2014, report 2010.

6. Jelsbak $L$, Johansen HK, Frost $A L$, et al. Molecular epidemiology and dynamics of Pseudomonas aeruginosa populations in lungs of cystic fibrosis patients. Infect Immun 2007;75(5):2214-24.

7. Johansen HK, Aanaes K, Pressler T, et al. Colonisation and infection of the paranasal sinuses in cystic fibrosis patients is accompanied by a reduced PMN response. J Cyst Fibros 2012;11(6):525-31.

8. Marvig RL, Sommer LM, Molin S, Johansen HK. Convergent evolution and adaptation of Pseudomonas aeruginosa within patients with cystic fibrosis. Nat Genet. 2015;47(1):57-64

9. Mainz JG, Naehrlich L, Schien $M$, et al.
Concordant genotype of upper and lower airways $P$ aeruginosa and $S$ aureus isolates in cystic fibrosis. Thorax 2009;64(6):535-40.

10. Illing EA, Woodworth BA. Management of the upper airway in cystic fibrosis. Curr Opin Pulm Med 2014;20(6):623-31.

11. Bonestroo HJ, de Winter-de Groot KM, van der Ent CK, Arets HG. Upper and lower airway cultures in children with cystic fibrosis: do not neglect the upper airways. J Cyst Fibros 2010;9(2):130-4

12. Berkhout MC, Rijntjes E, El Bouazzaoui LH, Fokkens WJ, Brimicombe RW, Heijerman HG. Importance of bacteriology in upper airways of patients with Cystic Fibrosis. J Cyst Fibros 2013;12(5):525-9.

13. Hansen SK, Rau MH, Johansen HK et al. Evolution and diversification of Pseudomonas aeruginosa in the paranasal sinuses of cystic fibrosis children have implications for chronic lung infection. ISME J 2012;6(1):31-45.

14. Mainz JG, Hentschel J, Schien C, et al. Sinonasal persistence of Pseudomonas aeruginosa after lung transplantation. J Cyst Fibros 2012;11(2):158-61.

15. Vital D, Hofer M, Benden C, Holzmann D, Boehler A. Impact of sinus surgery on pseudomonal airway colonization, bronchiolitis obliterans syndrome and survival in cystic fibrosis lung transplant recipients. Respiration 2013;86(1):25-31.

16. Berkhout MC, van Rooden CJ, Rijntjes $E_{\text {, }}$ Fokkens WJ, Bouazzaoui LH, Heijerman HG. Sinonasal manifestations of cystic fibrosis: a correlation between genotype and pheno- type? J Cyst Fibros 2014;13(4):442-8.

17. Eggesbo HB, Sovik S, Dolvik S, Kolmannskog F. CT characterization of inflammatory paranasal sinus disease in cystic fibrosis. Acta Radiol 2002;43(1):21-28.

18. Habib AR, Buxton JA, Singer J, Wilcox PG, Javer AR, Quon BS. Association between Chronic Rhinosinusitis and Health-Related Quality of Life in Adults with Cystic Fibrosis. Ann Am Thorac Soc 2015; 12(8):1163-9.

19. Doth F, Hentschel J, Fischer N, et al. Reduced effect of intravenous antibiotic treatment on sinonasal markers in pulmonary inflammation. Rhinology 2015;53(3):249-59.

20. Hopkins C, Gillett S, Slack R, Lund VJ, Browne JP. Psychometric validity of the 22-item Sinonasal Outcome Test. Clin Otolaryngol 2009;34(5):447-54.

21. Quittner AL, Buu A, Messer MA, Modi AC, Watrous M. Development and validation of The Cystic Fibrosis Questionnaire in the United States: a health-related qualityof-life measure for cystic fibrosis. Chest 2005;128(4):2347-54.

22. Aanaes K, Johansen HK, Skov M, et al. Clinical effects of sinus surgery and adjuvant therapy in cystic fibrosis patients - can chronic lung infections be postponed? Rhinology 2013;51(3):222-30.

23. Koch C, Høiby N. Diagnosis and treatment of cystic fibrosis. Respiration 2000;67(3):23947.

24. Fokkens WJ, Lund VJ, Mullol J, et al. EPOS 2012: European position paper on rhinosinusitis and nasal polyps 2012. A summa- 
ry for otorhinolaryngologists. Rhinology 2012;50(1):1-12

25. Proesmans M, Balinska-Miskiewicz W, Dupont L, et al. Evaluating the "Leeds criteria" for Pseudomonas aeruginosa infection in a cystic fibrosis centre. Eur Respir J 2006;27(5):937-43.

26. Johansen HK, Moskowitz SM, Ciofu O, Pressler T, Høiby N. Spread of colistin resistant non-mucoid Pseudomonas aeruginosa among chronically infected Danish cystic fibrosis patients. J Cyst Fibros 2008;7(5):3917.

27. Taylor-Robinson D, Whitehead M, Diderichsen $F$, et al. Understanding the natural progression in \%FEV1 decline in patients with cystic fibrosis: a longitudinal study. Thorax 2012;67(10):860-6.

28. Høiby N, Flensborg EW, Beck B, Friis B, Jacobsen SV, Jacobsen L. Pseudomonas aeruginosa infection in cystic fibrosis. Diagnostic and prognostic significance of Pseudomonas aeruginosa precipitins determined by means of crossed immunoelectrophoresis. Scand J Respir Dis 1977;58(2):65-79.

29. Høiby N, Koch C. Maintenance treatment of chronic pseudomonas aeruginosa infection in cystic fibrosis. Thorax 2000;55(5):349-50,

30. Tenover FC, Arbeit RD, Goering RV, et al. Interpreting chromosomal DNA restriction patterns produced by pulsed-field gel electrophoresis: criteria for bacterial strain typ- ing. J Clin Microbiol 1995;33(9):2233-9.

31. Taylor RJ, Miller JD, Rose AS, et al. Comprehensive quality of life outcomes for pediatric patients undergoing endoscopic sinus surgery. Rhinology 2014;52(4):327-33.

32. Mainz JG, Koitschev A. Pathogenesis and management of nasal polyposis in cystic fibrosis. Curr Allergy Asthma Rep 2012;12(2):163-74.

33. Cornet ME, Georgalas C, Reinartz SM Fokken WJ. Long-term results of functional endoscopic sinus surgery in children with chronic rhinosinusitis with nasal polyps. Rhinology 2013;51(4):328-34.

34. Taccetti G, Campana S, Festini F, Mascherini M, Döring G. Early eradication therapy against Pseudomonas aeruginosa in cystic fibrosis patients. Eur Respir J 2005;26(3):45861.

35. Virgin FW, Rowe SM, Wade MB, et al. Extensive surgical and comprehensive postoperative medical management for cystic fibrosis chronic rhinosinusitis. Am J Rhinol Allergy 2012;26(1):70-5.

36. Umetsu DT, Moss RB, King VV, Lewiston NJ. Sinus disease in patients with severe cystic fibrosis: relation to pulmonary exacerbation. Lancet 1990;335(8697):1077-8.

37. Holzmann D, Speich R, Kaufmann $T$, et al. Effects of sinus surgery in patients with cystic fibrosis after lung transplantation: a 10-year experience. Transplantation 2004;77(1):134-6.
38. Aanaes K, Johansen HK, Poulsen SS, Pressler T, von Buchwald C, Høiby N. Secretory IgA as a diagnostic tool for Pseudomonas aeruginosa respiratory colonization. J Cyst Fibros 2013;12(1):81-7.

39. Alanin MC, Johansen HK, Aanaes K, et al. Simultaneous sinus and lung infections in patients with primary ciliary dyskinesia. Acta Otolaryngol. 2015;135(1):58-63.

Mikkel Christian Alanin, M.D

Department of Otorhinolaryngo-

logy - Head and Neck Surgery and

Audiology

Copenhagen University Hospital

Rigshospitalet

Blegdamsvej 9

2100 Copenhagen

Denmark

Telephone: +45-3-545 2071

Fax: +45-3-545 2629

E-mail: mikkel.christian.alanin.01@ regionh.dk

\section{ADVERTISEMENT}

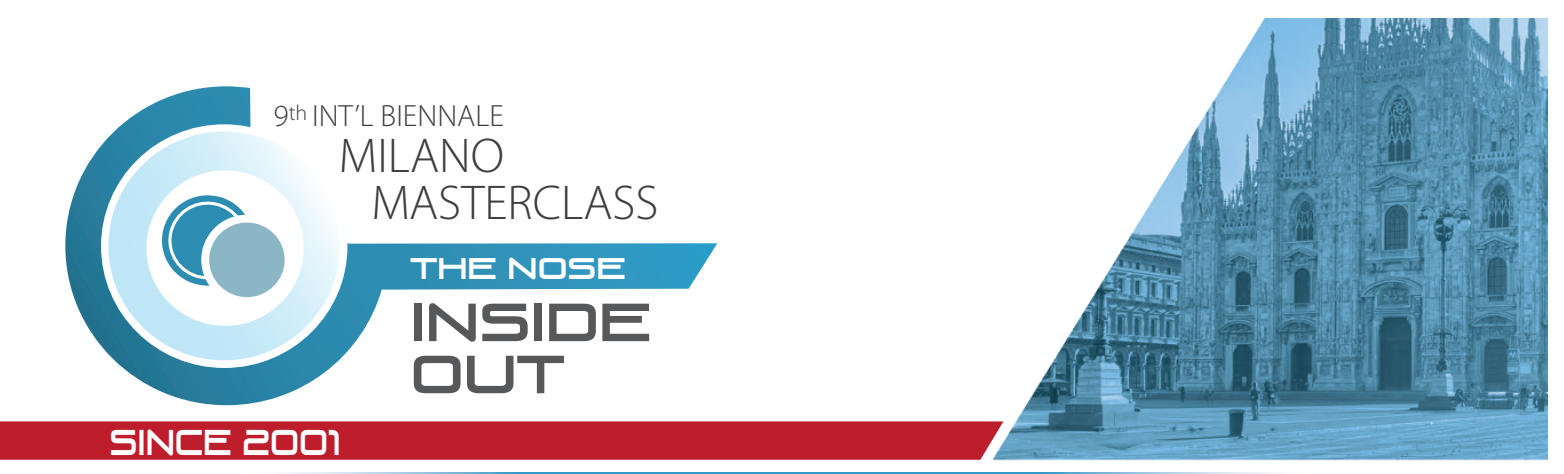

Directors: Paolo Castelnuovo \& Pietro Palma

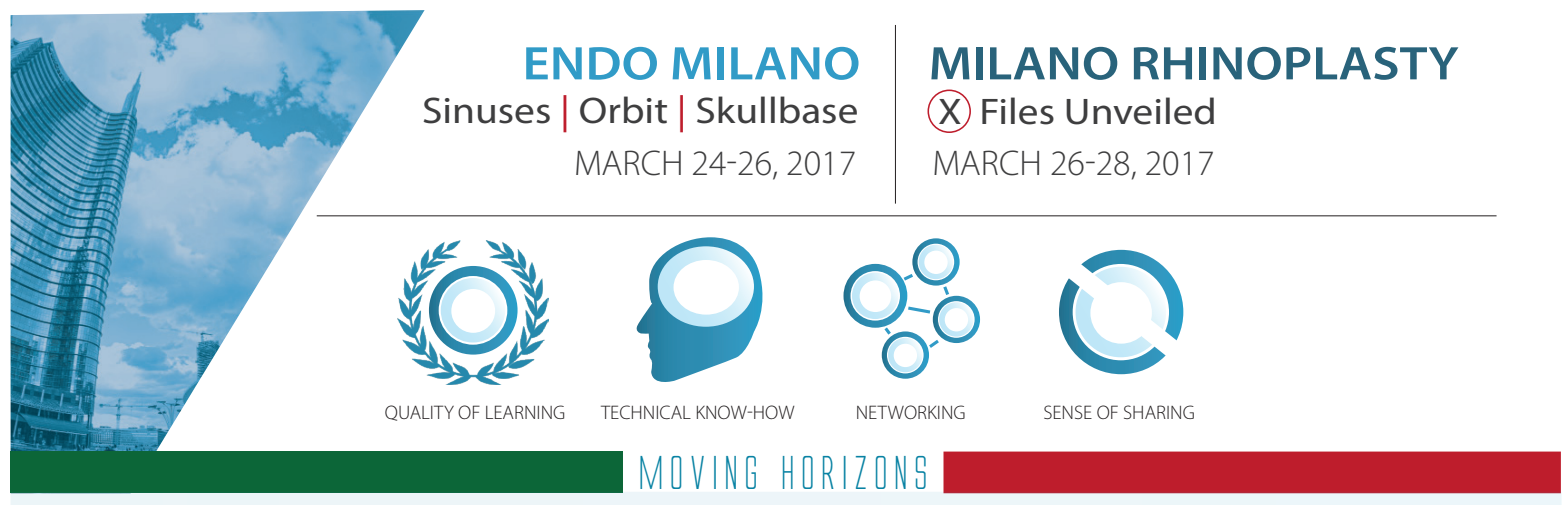

MILANO, ITALY | MARCH 24-28, 2017

www.milanomasterclass.it 